\title{
Plasma Concentrations of Monocyte Chemoattractant Protein 1 (MCP-1) and Neopterin in the Coronary Circulation of Patients With Coronary Artery Disease
} Association With the Severity of Coronary Atherosclerosis

\author{
Takayuki Tanaka, MD; Yuichi Nakamura, MD; Akimitsu Nasuno, MD; Tohru Mezaki, MD; \\ Koutarou Higuchi, MD; Hiroshi Fukunaga, MD; Keiichi Tsuchida, MD; Kazuyuki Ozaki, MD; \\ Tomoyuki Hori, MD*; Taku Matsubara, MD**; Yoshifusa Aizawa, MD
}

\begin{abstract}
Background The dynamics of MCP-1 and neopterin and the relation between their concentrations in coronary circulation and the severity of coronary atherosclerosis were evaluated in patients with stable coronary artery disease $(\mathrm{CAD})$.

Methods and Results Blood samples were obtained from the aortic root (Ao) and coronary sinus (CS) of 78 patients who underwent coronary angiography. Plasma MCP-1 and neopterin concentrations were measured using an enzyme-linked immunosorbent assay method and the CS-Ao differences were calculated. The severity of coronary heart disease (CHD) was evaluated in 52 patients who had no history of coronary angioplasty, using 3 coronary scoring systems: the clinical 1- to 3-vessel disease score, the American Heart Association extension score (1-15 segments), and the Gensini score. The plasma MCP-1 and neopterin concentrations increased significantly with age. The CS-Ao differences for neopterin showed weak, but significant, positive correlation with the Gensini score $(\mathrm{r}=0.347, \mathrm{p}=0.013)$. There were no correlations among the MCP- 1 concentrations in the Ao or $\mathrm{CS}$, or in the CS-Ao difference, with the severity of CHD.

Conclusions The results indicate that neopterin is a useful marker of the severity of coronary atherosclerosis in patients with stable CAD, acting as an index of the activity of monocytes/macrophages. (Circ J 2004; 68: 114120)
\end{abstract}

Key Words: Atherosclerosis; Coronary artery disease; Monocyte chemoattractant protein-1 (MCP-1); Neopterin

$\mathbf{I}$ nflammatory cytokines are involved in the pathogenesis of atherosclerosis by promoting the migration of inflammatory cells. Monocyte chemoattractant protein-1 (MCP-1) has been detected in atherosclerotic lesions using anti-MCP-1 antibody ${ }^{1}$ and by in situ hybridization? 2,3 MCP-1 mRNA expression has been detected in endothelial cells, vascular smooth muscle cells and macrophages in atherosclerotic lesions, 4 and monocytes are attracted to the atherosclerotic lesions? 5 Oxidized low-density lipoprotein (Ox-LDL) accumulates within these monocytes and macrophages leading to the formation and progression of atherosclerosis6 Moreover, in apolipoprotein E-deficient mice MCP-1 expression by macrophages accelerates that progression by increasing both the number of macrophages and the accumulation of Ox-LDL7,8 It has been recently suggested that MCP-1 directly stimulates the proliferation and migration of vascular smooth muscle cells, and that deletion of the CCR2 gene (a receptor for MCP-1) partially suppresses atherosclerotic lesion development in apolipo-

(Received June 16, 2003; revised manuscript received October 23, 2003; accepted November 7, 2003)

Division of Cardiology, Niigata University Graduate School of Medical \& Dental Sciences, *Dr Hori's office, and **Division of Cardiology, Shinrakuen Hospital, Niigata, Japan

Mailing address: Takayuki Tanaka, MD, Division of Cardiology, Niigata University Graduate School of Medical \& Dental Sciences, 1-

754 Asahi-machi-dori, Niigata 951-8510, Japan protein E-deficient mice by inhibiting monocyte recruitment.$^{10}$ In contrast, neopterin, a byproduct of the guanosine triphosphate pathway, is produced by activated monocytes/macrophages after stimulation by interferon- (INF) gamma!1 There is also a close relation between the concentration of neopterin produced by these cells and their capacity to release hydrogen peroxide; ${ }^{12}$ that is, the concentration reflects the level of oxidative stress caused by activation of the immune system and therefore, neopterin serves as a marker of the activation status of these cells! ${ }^{13}$

Circulating concentrations of MCP-1 and neopterin are reported to be elevated in patients with acute coronary syndrome. However, the concentrations in patients with stable angina pectoris remain uncertain because measurements in the peripheral blood do not differ significantly. In the present study, we obtained blood samples simultaneously from the aortic root (Ao) and coronary sinus (CS), and investigated whether plasma concentrations of these markers reflect the severity of coronary atherosclerosis in patients with stable coronary artery disease (CAD).

\section{Methods}

Patients

Seventy-eight patients undergoing cardiac catheterization for proven or clinically suspected CAD were recruited for this study (63 men, 15 women; mean age $62 \pm 11$ years, 
range 34-78 years). The exclusion criteria were acute myocardial infarction within the previous 2 weeks, unstable angina pectoris, New York Heart Association class III or IV congestive heart failure or a left ventricular ejection fraction $<25 \%$, acute infection, acute state of a chronic infectious or inflammatory disease, neoplasm 14 hematologic disorders, and acute or chronic liver or kidney disease. The study protocol was approved by the ethics committee of Niigata University Medical Hospital, and written informed consent was obtained from each patient before participation. The patients' characteristics were obtained from medical records with regard to history of hypertension, hypercholesterolemia, diabetes, history of smoking, previous myocardial infarction, and prior coronary intervention. Patients considered to have arterial hypertension had a current systolic/diastolic blood pressure $>140 / 90 \mathrm{mmHg}$ or were receiving antihypertensive therapy. Patients with hypercholesterolemia were defined as those having a total-cholesterol $>220 \mathrm{mg} / \mathrm{dl}$ or who were receiving lipid lowering therapy. Diabetes was defined using the American Diabetes Association criteria! ${ }^{15}$ Body mass index (BMI) was calculated as weight $(\mathrm{kg}) /$ height $(\mathrm{m})^{2}$.

\section{Coronary Angiography and Blood Sampling}

In all patients, coronary angiography was performed using the Judkins technique with a 6F catheter (Johnson \& Johnson, Miami Lakes, FL, USA). Before coronary angiography, the Judkins catheter was introduced via the femoral artery and advanced into the ascending aorta, and a $7 \mathrm{~F}$ Simmons catheter (Hanako-Medical Co, Urawa, Japan) was inserted through the femoral vein and advanced into the CS. Blood samples were then obtained simultaneously from the Ao and CS 16,17

\section{Determining the Severity of Coronary Atherosclerosis}

We used 3 different coronary scores to evaluate the angiographic severity and extension of CAD in 52 patients without prior coronary intervention. First, patients were classified according to the number of stenosed ( $>50 \%$ of luminal diameter) or occluded (1- to 3-vessel disease) vessels; second, a quantitative extension score (1-15 segments) using the American Heart Association criteria was calculated; and third, a qualitative and quantitative evaluation was performed using the Gensini score (GS) 18 The GS defines narrowing of the lumen of the coronary arteries as 1 for 1-25\% narrowing, 2 for 26-50\% narrowing, 4 for 51$75 \%$ narrowing, 8 for $76-90 \%$ narrowing, 16 for $91-99 \%$
Table 1 Baseline Characteristics

\begin{tabular}{lc}
\hline \hline & $n=78$ \\
Male gender & $63(81 \%)$ \\
Age (mean \pm SD) & $61.9 \pm 11.0$ \\
Hypertension & $43(55 \%)$ \\
Hypercholesterolemia & $42(54 \%)$ \\
Diabetes mellitus & $34(44 \%)$ \\
History of smoking & $54(69 \%)$ \\
Obesity (BMI >24) & $32(41 \%)$ \\
Family history of coronary heart disease & $21(27 \%)$ \\
Previous myocardial infarction & $24(31 \%)$ \\
History of coronary angioplasty & $26(33 \%)$ \\
\hline
\end{tabular}

BMI, body mass index.

narrowing, and 32 for total occlusion. The score is then multiplied by a factor that incorporates the importance of the lesion's position in the coronary arterial tree; for example, 5 for the left main coronary artery, 2.5 for the proximal left anterior descending artery (LAD) or proximal left circumflex artery (LCX), 1.5 for the mid-region of the $\mathrm{LAD}$, and 1 for the distal LAD or mid-distal region of the LCx. Observers who were unaware of the clinical and laboratory data scored all the coronary angiograms.

\section{Measuring MCP-1 and Neopterin Concentrations}

Plasma was separated from the blood samples by centrifugation and stored at $-40^{\circ} \mathrm{C}$ until required for the MCP-1 and neopterin assays. MCP-1 and neopterin concentrations were measured using an enzyme-linked immunosorbent assay kit (MCP-1: R\&D Systems, Minneapolis, MN, USA; Neopterin: IBL, Hamburg, Germany). All assays were performed in duplicate. The minimum detectable doses of MCP-1 and neopterin are typically less than $5.0 \mathrm{pg} / \mathrm{ml}$ and $0.18 \mathrm{ng} / \mathrm{ml}$. The intra-assay coefficient of variation of the kit at the MCP-1 antigen concentration of $364 \mathrm{pg} / \mathrm{ml}$ was $4.7 \%$ and $5.5 \%$ at the neopterin antigen concentration of $1.93 \mathrm{ng} / \mathrm{ml}$. The inter-assay coefficient of variation of the kit at the MCP-1 antigen concentration of $352 \mathrm{pg} / \mathrm{ml}$ was $5.8 \%$ and $10.3 \%$ at the neopterin antigen concentration of $1.85 \mathrm{ng} / \mathrm{ml}$.

\section{Data Analysis}

Plasma MCP-1 and neopterin concentrations were measured for both the Ao and CS, and the differences between the samples (CS-Ao) were calculated. We compared their concentrations with clinical characteristics, including age, gender, coronary risk factors, history of previous myocar-
[A]

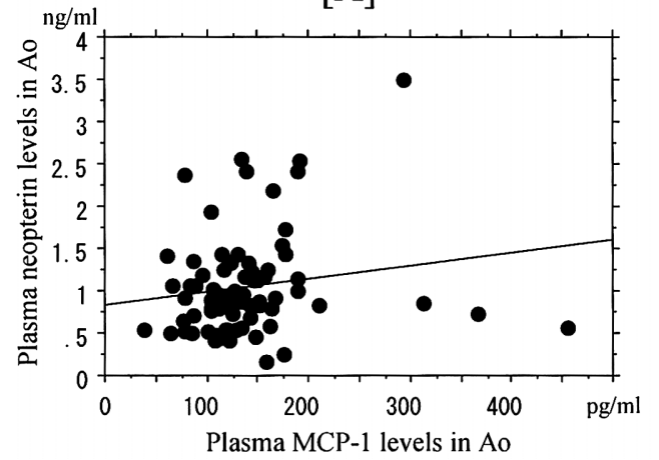

[B]

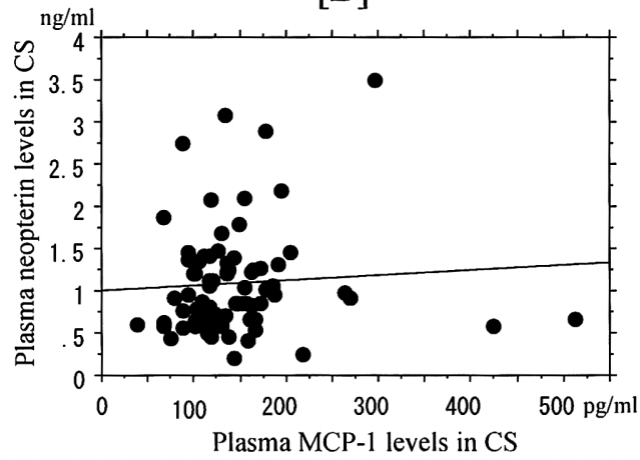

Fig 1. Relation between the plasma MCP-1 and neopterin concentrations in (A) the aortic root (Ao) and (B) the coronary sinus (CS). 
Table 2 Baseline Characteristics and Concentrations of MCP-1 and Neopterin

\begin{tabular}{|c|c|c|c|c|c|c|}
\hline & $\begin{array}{l}M C P-1(\mathrm{Ao}) \\
\quad(\mathrm{pg} / \mathrm{ml})\end{array}$ & $\begin{array}{l}M C P-1(C S) \\
\quad(\mathrm{pg} / \mathrm{ml})\end{array}$ & $\begin{array}{l}M C P-1(\Delta) \\
\quad(p g / m l)\end{array}$ & $\begin{array}{c}\text { Neopterin }(\mathrm{Ao}) \\
(\mathrm{ng} / \mathrm{ml})\end{array}$ & $\begin{array}{c}\text { Neopterin }(C S) \\
(\mathrm{ng} / \mathrm{ml})\end{array}$ & $\begin{array}{c}\text { Neopterin }(\Delta) \\
\quad(\mathrm{ng} / \mathrm{ml})\end{array}$ \\
\hline \multicolumn{7}{|c|}{ Gender } \\
\hline (M) & $130.6 \pm 62.5$ & $137.6 \pm 72.8$ & $7.1 \pm 23.7$ & $0.988 \pm 0.523$ & $1.032 \pm 0.557$ & $0.044 \pm 0.221$ \\
\hline$(F)$ & $156.4 \pm 77.0$ & $157.9 \pm 70.0$ & $1.6 \pm 17.6$ & $1.262 \pm 0.853$ & $1.285 \pm 0.882$ & $0.024 \pm 0.314$ \\
\hline \multicolumn{7}{|c|}{ Hypertension } \\
\hline$(+)$ & $135.6 \pm 68.3$ & $140.5 \pm 65.7$ & $4.8 \pm 15.3$ & $1.154 \pm 0.729$ & $1.202 \pm 0.767$ & $0.049 \pm 0.273$ \\
\hline$(-)$ & $135.4 \pm 63.5$ & $142.8 \pm 80.5$ & $7.4 \pm 29.5$ & $0.900 \pm 0.359$ & $0.929 \pm 0.366$ & $0.029 \pm 0.181$ \\
\hline \multicolumn{7}{|c|}{ Hypercholesterolemia } \\
\hline$(+)$ & $126.2 \pm 50.5$ & $129.7 \pm 48.2$ & $3.5 \pm 14.1$ & $1.015 \pm 0.607$ & $1.032 \pm 0.673$ & $0.017 \pm 0.257$ \\
\hline$(-)$ & $146.4 \pm 79.3$ & $155.3 \pm 91.7$ & $8.9 \pm 29.7$ & $1.071 \pm 0.609$ & $1.137 \pm 0.591$ & $0.066 \pm 0.220$ \\
\hline \multicolumn{7}{|c|}{ Diabetes mellitus } \\
\hline$(+)$ & $120.7 \pm 41.6$ & $123.8 \pm 43.3$ & $3.1 \pm 13.0$ & $1.006 \pm 0.524$ & $1.087 \pm 0.590$ & $0.081 \pm 0.269$ \\
\hline$(-)$ & $147.0 \pm 78.2$ & $155.2 \pm 86.4$ & $8.2 \pm 27.9$ & $1.068 \pm 0.663$ & $1.077 \pm 0.672$ & $0.009 \pm 0.213$ \\
\hline \multicolumn{7}{|c|}{ History of smoking } \\
\hline$(+)$ & $128.0 \pm 67.6$ & $134.9 \pm 77.4$ & $7.0 \pm 23.0$ & $0.966 \pm 0.519$ & $1.004 \pm 0.576$ & $0.038 \pm 0.209$ \\
\hline$(-)$ & $152.5 \pm 59.3$ & $156.4 \pm 57.8$ & $3.8 \pm 22.2$ & $1.208 \pm 0.746$ & $1.212 \pm 0.732$ & $0.044 \pm 0.302$ \\
\hline \multicolumn{7}{|c|}{ Obesity $(B M I>24)$} \\
\hline$(+)$ & $139.9 \pm 81.7$ & $148.2 \pm 94.7$ & $8.3 \pm 28.5$ & $0.941 \pm 0.538$ & $0.907 \pm 0.511$ & $-0.034 \pm 0.209$ \\
\hline$(-)$ & $132.5 \pm 52.7$ & $136.9 \pm 51.9$ & $4.4 \pm 17.7$ & $1.109 \pm 0.642$ & $1.199 \pm 0.686$ & $0.090 \pm 0.248$ \\
\hline \multicolumn{7}{|c|}{ Family history of coronary heart disease } \\
\hline$(+)$ & $118.7 \pm 42.4$ & $121.7 \pm 43.8$ & $3.0 \pm 14.4$ & $1.049 \pm 0.604$ & $1.080 \pm 0.606$ & $0.032 \pm 0.262$ \\
\hline$(-)$ & $141.7 \pm 71.8$ & $148.8 \pm 79.3$ & $7.1 \pm 25.1$ & $1.039 \pm 0.610$ & $1.082 \pm 0.649$ & $0.043 \pm 0.234$ \\
\hline \multicolumn{7}{|c|}{ Previous MI } \\
\hline$(+)$ & $133.4 \pm 54.1$ & $139.7 \pm 56.6$ & $6.4 \pm 13.0$ & $1.116 \pm 0.769$ & $1.226 \pm 0.758$ & $0.110 \pm 0.230$ \\
\hline$(-)$ & $136.5 \pm 70.8$ & $142.3 \pm 78.7$ & $5.8 \pm 26.0$ & $1.008 \pm 0.519$ & $1.016 \pm 0.565$ & $0.008 \pm 0.239$ \\
\hline
\end{tabular}

Values are expressed as mean $\pm S D$.

BMI, body mass index; Previous MI, previous myocardial infarction; Ao, aortic root; CS, coronay sinus.

*No associations between the plasma MCP-1 and neopterin concentrations nor between the other baseline characteristics of the patients.

[A]

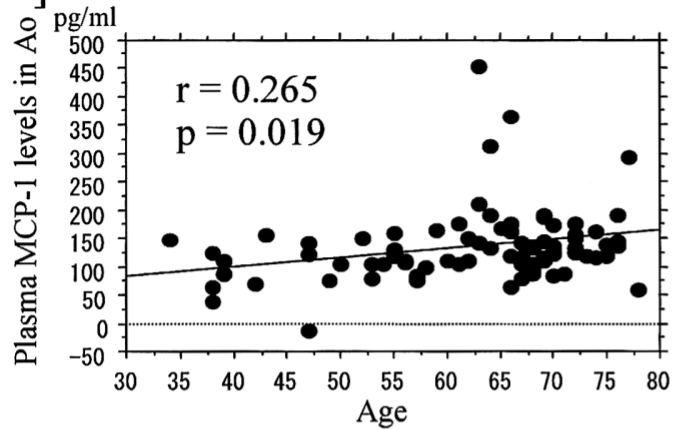

[C]

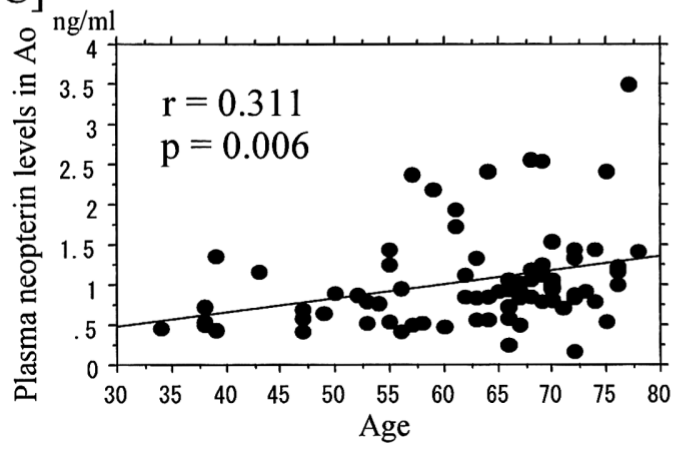

[B]

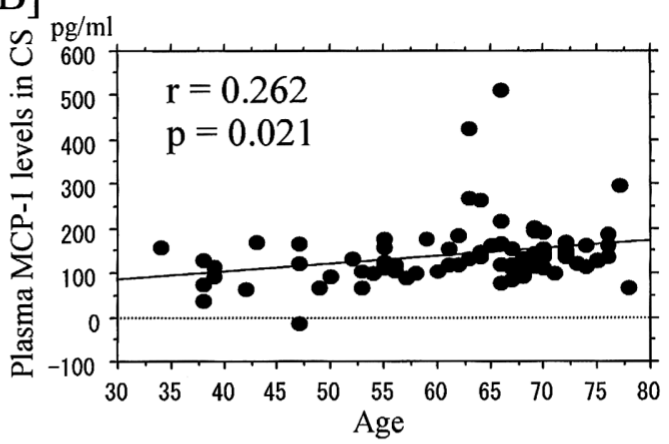

[D]

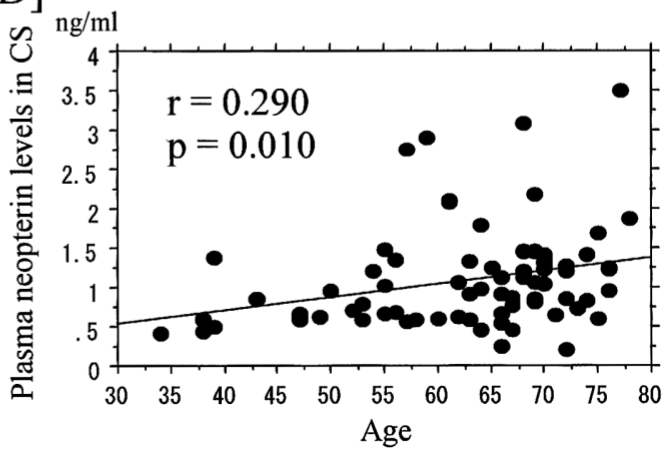

Fig 2. Correlation between age and the plasma concentrations of MCP-1 in the aortic root (Ao) (A) and coronary sinus (CS) (B) and neopterin in the Ao (C) and CS (D).

dial infarction, prior coronary intervention, and laboratory data. Moreover, in the patients who had no history of coronary intervention, we examined the association between the markers and the severity of CAD as defined by the coro- nary scoring systems. Where we refer to the CS-Ao difference, the coronary score is expressed as the sum of the values for the left coronary arteries only. 
Table 3 Mean Concentrations of MCP-1 and Neopterin in Relation to Coronary Scores

\begin{tabular}{|c|c|c|c|c|c|c|c|c|c|c|}
\hline & No. & $\begin{array}{l}M C P-1(\mathrm{Ao}) \\
\quad(\mathrm{pg} / \mathrm{ml})\end{array}$ & $\begin{array}{l}M C P-1(C S) \\
\quad(\mathrm{pg} / \mathrm{ml})\end{array}$ & No. * & $\begin{array}{l}M C P-1(\Delta) * \\
\quad(p g / m l)\end{array}$ & No. & $\begin{array}{l}\text { Neopterin }(A o) \\
(\mathrm{ng} / \mathrm{ml})\end{array}$ & $\begin{array}{c}\text { Neopterin }(C S) \\
(\mathrm{ng} / \mathrm{ml})\end{array}$ & No.* & $\begin{array}{l}\text { Neopterin }(\Delta) * \\
(n g / m l)\end{array}$ \\
\hline \multicolumn{11}{|l|}{ Clinical score } \\
\hline 0 -vessel disease & 28 & $151.8 \pm 88.9$ & $157.3 \pm 98.5$ & 32 & $7.7 \pm 33.2$ & 28 & $1.017 \pm 0.558$ & $1.031 \pm 0.508$ & 32 & $0.010 \pm 0.249$ \\
\hline 1-vessel disease & 10 & $141.5 \pm 32.5$ & $150.7 \pm 48.5$ & 11 & $0.9 \pm 9.1$ & 10 & $0.934 \pm 0.542$ & $0.985 \pm 0.720$ & 11 & $0.121 \pm 0.287$ \\
\hline 2-vessel disease & 7 & $150.8 \pm 74.0$ & $152.6 \pm 75.4$ & 9 & $2.3 \pm 7.2$ & 7 & $1.195 \pm 1.037$ & $1.312 \pm 1.035$ & 8 & $0.167 \pm 0.239$ \\
\hline 3-vessel disease & 7 & $112.8 \pm 47.6$ & $115.2 \pm 50.3$ & & & 6 & $1.226 \pm 0.727$ & $1.439 \pm 0.941$ & & \\
\hline \multicolumn{11}{|l|}{ Extension score } \\
\hline 0 segments & 28 & $151.8 \pm 88.9$ & $157.3 \pm 98.5$ & 32 & $7.7 \pm 33.2$ & 28 & $1.017 \pm 0.558$ & $1.031 \pm 0.508$ & 32 & $0.010 \pm 0.249$ \\
\hline $1-3$ segments & 17 & $145.3 \pm 51.7$ & $151.5 \pm 58.8$ & 11 & $0.7 \pm 7.9$ & 17 & $1.041 \pm 0.766$ & $1.120 \pm 0.849$ & 11 & $0.119 \pm 0.269$ \\
\hline 4 segments & 7 & $112.8 \pm 47.6$ & $115.2 \pm 50.3$ & 9 & $6.2 \pm 9.6$ & 6 & $1.226 \pm 0.727$ & $1.439 \pm 0.941$ & 8 & $0.257 \pm 0.224$ \\
\hline
\end{tabular}

Values are expressed as mean $\pm S D$. Ao, aortic root; $C S$, coronay sinus.

* Mean concentrations for plasma MCP-1 and neopterin in the Ao and the CS are for both the right and left coronary arteries, but in the CS-Ao differences are for the left coronary artery only.
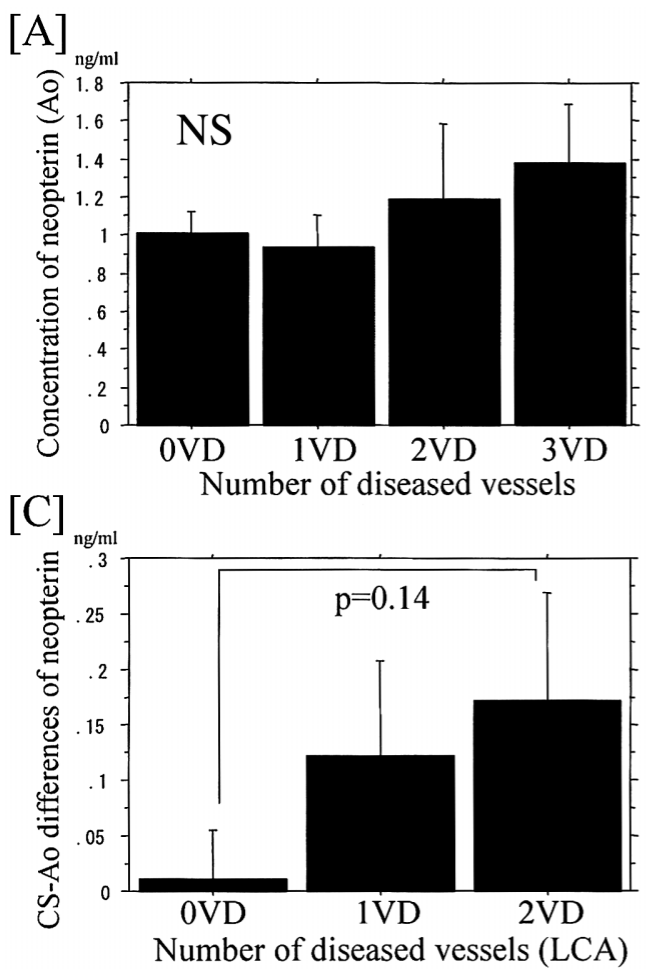

[B]

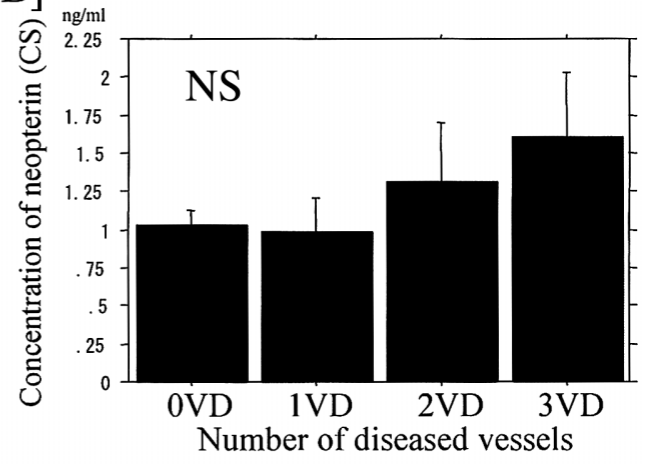

Fig 3. Mean plasma neopterin concentrations in the aortic root (Ao) (A) and coronary sinus (CS) (B) of patients with novessel disease (0VD), 1-vessel disease (1VD), 2-vessel disease (2VD), and 3-vessel disease (3VD). The CS-Ao difference in the neopterin concentrations $(\mathrm{C})$ in patients with $0 \mathrm{VD}, 1 \mathrm{VD}$, and $2 \mathrm{VD}$ for the left coronary arteries (LCA) only.

\section{Statistical Analysis}

Data are presented as the mean value \pm standard deviation (SD). Comparisons of the data between groups were analyzed using Student's unpaired t-test. Comparisons of the data among 3 groups, the number of stenosed or occluded vessels, and the quantitative extension score were performed using one-way ANOVA followed by Bonferroni's correction for multiple comparisons. Linear regression analysis was used to determine whether there were any correlations between MCP-1 and neopterin and age and GS. A value of $\mathrm{p}<0.05$ was considered significant.

\section{Results}

\section{Patient Characteristics}

Patient characteristics are summarized in Table 1. Fortythree $(55 \%)$ patients had hypertension, $42(54 \%)$ had hypercholesterolemia, 34 (44\%) had diabetes mellitus, 52 $(67 \%)$ had a history of smoking, $34(44 \%)$ were obese, 21 $(27 \%)$ had a family history of coronary heart disease, 24 (31\%) had a previous myocardial infarction, and $26(33 \%)$ had a history of coronary angioplasty. The mean left ventricular ejection fraction was $63.7 \pm 10.3 \%$, ranging from $40 \%$ to $89 \%$.

\section{Plasma MCP-1 and Neopterin Concentrations}

There were no associations between the plasma MCP-1 and neopterin concentrations nor between the other baseline characteristics of the patients (Fig 1, Table 2). The plasma MCP-1 concentrations in both the Ao $(r=0.265$, $\mathrm{p}=0.019)$ and CS $(r=0.262, p=0.021)$ increased significantly with age, as did the plasma neopterin concentrations in both the Ao $(r=0.311, p=0.006)$ and CS $(r=0.290, p=0.010)$ (Fig 2). 
[A]

A

$[\mathrm{C}]$

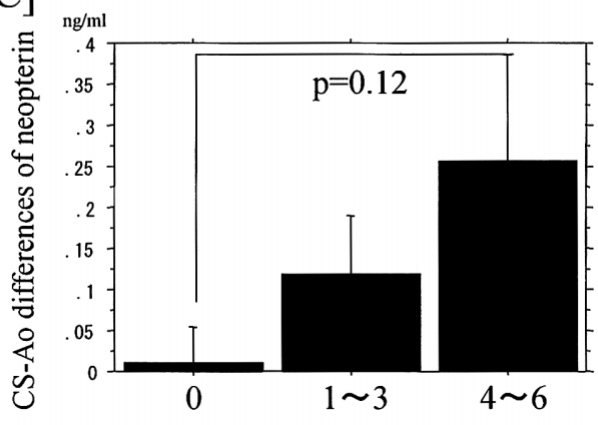

[B]

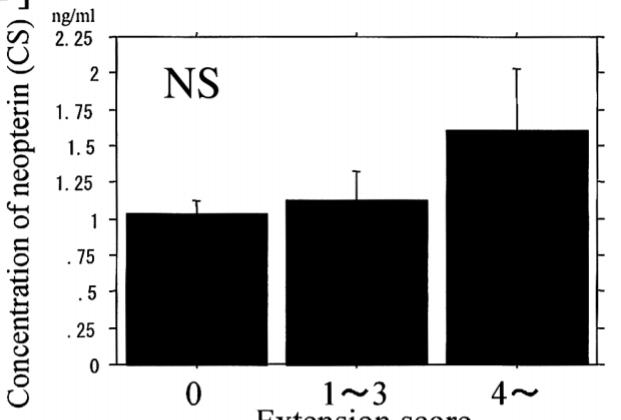

Extension score

Extension score

Fig 4. Mean plasma neopterin concentrations in the aortic root (Ao) (A) and coronary sinus (CS) (B) in patients with 0 segment (0), 1-3 segments (1-3) and 4+ segments (4+). The CS-Ao difference for neopterin (C) in patients with $0,1-3$, and $4+$ segments for the left coronary artery only.
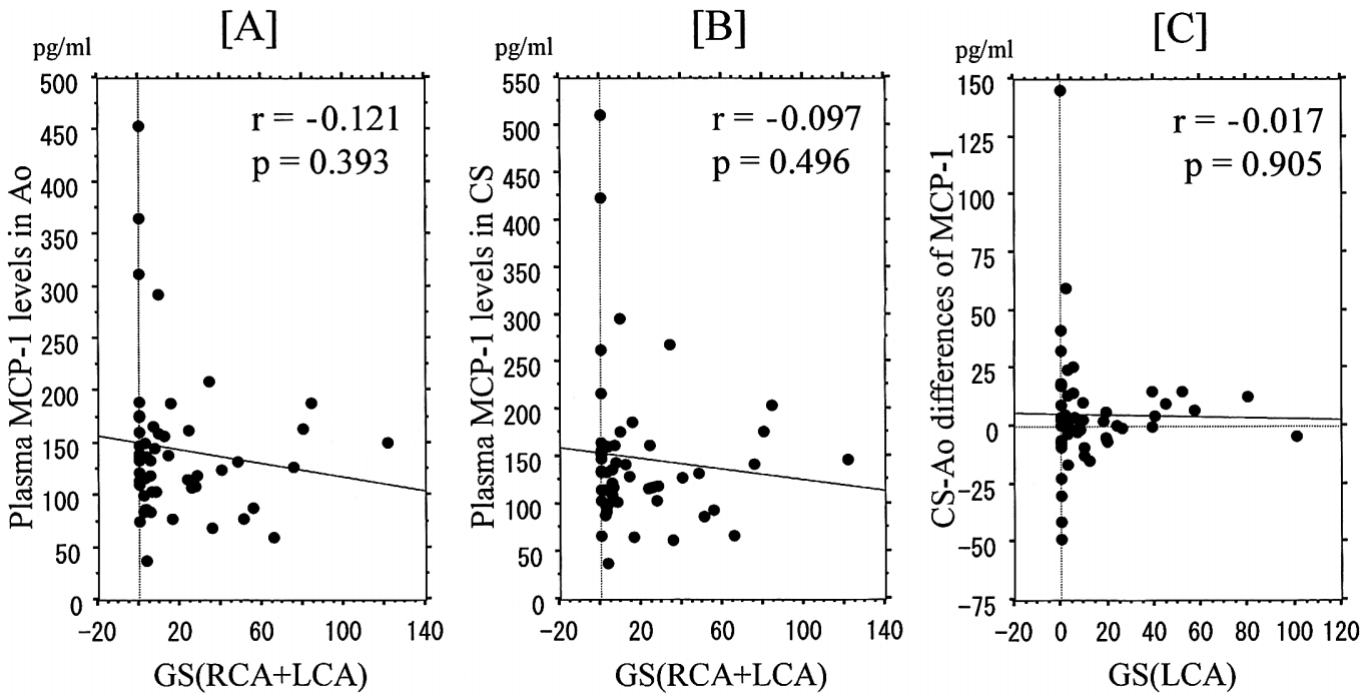

Fig 5. Relation between the plasma MCP-1 concentration in the aortic root (Ao) (A) and coronary sinus (CS) (B) and the severity of coronary atherosclerosis evaluated using the Gensini score (GS). The relation between the CS-Ao difference (C) for left coronary atherosclerosis evaluated using only the GS.

Correlations Among the Plasma MCP-1 Concentrations in the Ao and CS, and the CS-Ao Values and the Number of Vessels, Extension Score and GS

No association between the plasma MCP-1 concentrations and any coronary score was found (Table 3, Fig 5), nor was there any relation between severity or extension of coronary heart disease and MCP-1.
Correlations Among the Plasma Neopterin Concentrations in the Ao and CS, and CS-Ao Values and the Number of Vessels, Extension Score and GS

The plasma neopterin concentrations tended to increase with disease severity as evaluated by the number of vessels and the extension score, and this tendency became stronger with the $\mathrm{CS}-\mathrm{Ao}$ difference, but there were no significant differences among these concentrations in the Ao or CS or among the CS-Ao differences for neopterin in the 3 groups (Table 3, Figs 3,4). There was no correlation between the 

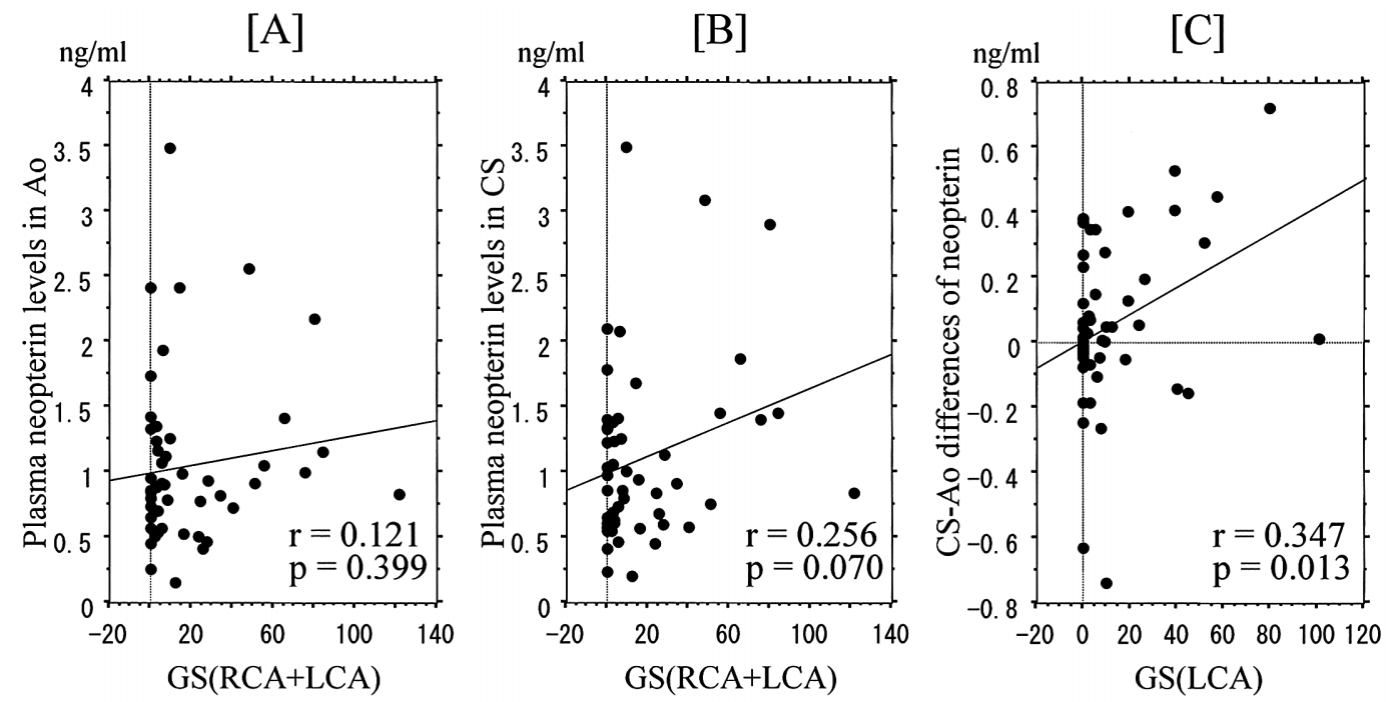

Fig 6. Relation between the plasma neopterin concentration in the aortic root (Ao) (A) and coronary sinus (CS) (B) and the severity of coronary atherosclerosis evaluated using the Gensini score (GS). The relation between the CS-Ao difference (C) for left coronary atherosclerosis evaluated using only the GS.

plasma neopterin concentrations in the Ao and CS and the GS (Ao: $\mathrm{r}=0.121, \mathrm{p}=0.399 ; \mathrm{CS}: \mathrm{r}=0.256, \mathrm{p}=0.070$ ), although there was a weak, but significant, correlation between the CS-Ao difference for neopterin and the GS $(\mathrm{r}=0.347, \mathrm{p}=0.013)($ Fig 6).

\section{Discussion}

We measured the plasma concentrations of MCP-1 and neopterin using blood samples obtained from the Ao and $\mathrm{CS}$, and assessed the relationships among these markers and the clinical characteristics, including the severity of coronary atherosclerosis, of patients with stable CAD.

The plasma concentrations of MCP-1 and neopterin were elevated significantly with age and the age-dependent rise might reflect the progression of systemic atherosclerosis. According to the results of our study, this hypothesis might apply to neopterin, but not necessarily to MCP-1. Nuclear factor (NF)-kappa B binding sites have been reported in the promoter of human MCP-1 gene! ${ }^{19}$ Therefore, MCP-1 expression might increase with age via the activation of NF-kappa B by the accumulated oxidant stress. A previous study reports that increased neopterin was demonstrated in renal dysfunction, 12 but in the present study, almost all plasma creatinine concentrations were normal $(0.756 \pm 0.183 \mathrm{mg} / \mathrm{dl}, 0.4-1.2 \mathrm{mg} / \mathrm{dl})$. Renal dysfunction was not the cause of age-dependent rise of neopterin in our study.

We also assessed the relationships among the plasma concentrations of MCP-1 and neopterin and the severity of coronary atherosclerosis. Previous studies suggest that peripheral blood concentrations of MCP-1 and neopterin increase in patients with acute coronary syndrome ${ }^{20-24}$ Coronary plaques of these patients have more extensive macrophage-rich areas than those with stable angina 25,26 Moreover, activated $\mathrm{T}$ cells contained in vulnerable plaques produce cytokine IFN-gamma, which activates systemic monocytes/macrophages. ${ }^{27,28}$ Consequently, systemic monocytes/macrophages and other cells may produce sufficient quantities of these factors to be detected in peripheral blood. Moreover, there might have been sufficient increases in these factors not to disappear in dispersion of the whole body burden of atherosclerotic tissue. Paradoxically, in acute coronary syndrome, none of these markers produced from systemic monocytes/macrophages and other cells can be estimated using peripheral blood, therefore the concentrations of these markers do not necessarily reflect the local state of coronary atherosclerosis.

We calculated the CS-Ao differences. We achieved sufficient sensitivity and assessed the specific production of these markers in coronary circulation. CS-Ao differences for MCP-1 showed no relation with the 3 coronary severity scores used in this study, whereas the CS-Ao differences for neopterin tended to increase with the severity of coronary atherosclerosis, and a significant positive correlation was found between CS-Ao differences for neopterin and the GS.

One possible explanation of this phenomenon is that the 2 markers reflect different aspects in the progression of atherosclerosis. MCP-1 is thought to affect the number of monocytes/macrophages attracted to the vessel wall. In acute coronary syndrome, vulnerable plaques clearly reflect increased numbers of macrophages and activated lymphocytes. ${ }^{29}$ MCP-1 might be a marker reflecting the instability of atherosclerotic lesions. On the other hand, neopterin production is more likely related to monocytes/macrophages activity than to increased numbers of these cells!1 There might be a so-called basal neopterin production following basal macrophage activity even in stable plaque. The quantity of neopterin might be determined by the total number of macrophages, which is proportional to the volume of atherosclerotic plaque. In stable angina, atherosclerotic lesions mainly consist of stable plaque and we might be able to ignore the aspect of monocytes/macrophages attraction to the vessel wall. This might be one of the reasons why the CS-Ao difference not for MCP-1 but for neopterin correlated with the severity of coronary atherosclerosis. Therefore, the CS-Ao difference for neopterin might be a useful parameter reflecting coronary plaque volume in patients with stable angina pectoris. 


\section{Study Limitations}

Firstly, we assessed the severity of coronary atherosclerosis using an angiography based scoring system. It is critical to estimate the total volume of coronary plaques to determine whether the CS-Ao difference for neopterin is a useful parameter for coronary plaque volume in patients with stable angina pectoris. All 3 scores used in this study determine disease severity by angiographic coronary stenosis, but do not evaluate plaque volume directly. Recently, an intravascular ultrasound system (IVUS) has been used to assess arterial plaque volume, but even that is unsuitable for assessing plaque volume throughout the coronary tree, including the distal lesions in smaller vessels. Because there is no method available for assessing the whole coronary plaque volume, we have to assess the severity of coronary disease angiographically. In future, novel imaging techniques such as multi-detector 3-dimensional computed tomography or high resolution magnetic resonance imaging may be employed for this purpose. Development of a novel method of measuring whole coronary plaque volume and further investigations are expected.

Secondly, this study is a cross-sectional study. Although we expect to predict future coronary events by evaluating these markers, we cannot generalize from the results of this study because the picture is of individual patients. Several studies have indicated that production of MCP-1 might be associated with the mechanisms of vascular remodeling after percutaneous transluminal coronary intervention 30,31 Follow-up studies are required to elucidate the relation among the production of MCP-1 and/or neopterin in coronary circulation and coronary events in the future.

Thirdly, the study comprised only a small number of patients.

\section{Conclusions}

Our findings suggest that neopterin, which is associated with the activity of monocytes/macrophages, is a useful marker for predicting the severity of coronary atherosclerosis in patients with stable CAD. Assessment of neopterin production in coronary circulation might be a useful indicator of the state and prognosis of coronary atherosclerosis.

\section{References}

1. Takeya M, Yoshimura T, Leonard EJ, Takahashi K. Detection of monocyte chemoattractant protein-1 in human atherosclerotic lesions by an anti-monocyte chemoattractant protein-1 monoclonal antibody. Hum Pathol 1993; 24: 534-539.

2. Nelken NA, Coughlin SR, Gordon D, Wilcox JN. Monocyte chemoattractant protein-1 in human atheromatous plaques. J Clin Invest 1991; 88: $1121-1127$.

3. Yla-Herttuala S, Lipton BA, Rosenfeld ME, Sarkioja T, Yoshimura $\mathrm{T}$, Leonard EJ, et al. Expression of monocyte chemoattractant protein-1 in macrophage-rich areas of human and rabbit atherosclerotic lesions. Proc Natl Acad Sci USA 1991; 88: 5252-5256.

4. Seino Y, Ikeda U, Takahashi M, Hojo Y, Irokawa M, Kasahara T, et al. Expression of monocyte chemoattractant protein-1 in vascular tissue. Cytokine 1995; 7: 575-579.

5. Jonasson L, Holm J, Skalli O, Bondjers G, Hansson GK. Regional accumulations of $\mathrm{T}$ cells, macrophages, and smooth muscle cells in the human atherosclerotic plaque. Arteriosclerosis 1986; 6: 131138.

6. Jennifer RH. The role of MCP-1 in atherosclerosis. Stem Cells 2000; 18: $65-66$

7. Aiello RJ, Bourassa PA, Lindsey S, Weng W, Natoli E, Rollins BJ, et al. Monocyte chemoattractant protein-1 accelerates atherosclerosis in apolipoprotein E-deficient mice. Arterioscler Thromb Vasc Biol 1999; 19: $1518-1525$.
8. Reckless J, Rubin EM, Verstuyft JB, Metcalfe JC, Grainger DJ. Monocyte chemoattractant protein-1 but not tumor necrosis factoralpha is correlated with monocyte infiltration in mouse lipid lesions. Circulation 1999; 99: 2310-2316.

9. Porreca E, Di Febbo C, Reale M, Castellani ML, Baccante G, Barbacane R, et al. Monocyte chemotactic protein 1 (MCP-1) is a mitogen for cultured rat vascular smooth muscle cells. $J$ Vasc Res 1997; 34: 58-65.

10. Boring L, Gosling J, Cleary M, Charo IF. Decreased lesion formation in CCR2-/- mice reveals a role for chemokines in the initiation of atherosclerosis. Nature 1998; 394: 894-897.

11. Huber C, Batchelor JR, Fuchs D, Hausen A, Lang A, Niederwieser $\mathrm{D}$, et al. Immune response-associated production of neopterin: Release from macrophages primarily under control of interferongamma. J Exp Med 1984; 160: 310-316.

12. Berdowska A, Zwirska-Korczala K. Neopterin measurement in clinical diagnosis. J Clin Pharm Ther 2001; 26: 319-329.

13. Neumann FJ, Marx N, Gawaz M, Brand K, Ott I, Rokitta C, et al. Induction of cytokine expression in leukocytes by binding of thrombin-stimulated platelets. Circulation 1997; 95: 2387-2394.

14. Zhang T, Koide N, Wada Y, Tsukioka K, Takayama K, Kono T, et al. Significance of monocyte chemotactic protein-1 and thymidine phosphorylase in angiogenesis of human cardiac myxoma. Circ J 2003; 67: 54-60.

15. Report of the Expert Committee on the Diagnosis and Classification of Diabetes Mellitus. Diabetes Care 1997; 20: 1183-1197.

16. Hori T, Matsubara T, Ishibashi T, Yamazoe M, Ida T, Higuchi K, et al. Decrease of nitric oxide end-products during coronary circulation reflects elevated basal coronary artery tone in patients with vasospastic angina. Jpn Heart J 2000; 41: 583-595.

17. Nasuno A, Matsubara T, Hori T, Higuchi K, Imai S, Nakagawa I, et al. Levels of soluble E-selectin and ICAM-1 in the coronary circulation of patients with stable coronary artery disease: Association with the severity of coronary atherosclerosis. Jpn Heart J 2002; 43: 93 101.

18. Gensini GG. A more meaningful scoring system for determining the severity of coronary heart disease. Am J Cardiol 1983; 51: 606.

19. Ueda A, Ishigatsubo Y, Okubo T, Yoshimura T. Transcriptional regulation of the human monocyte chemoattractant protein-1 gene: Cooperation of two NF-kappa B sites and NF-kappa B/Rel subunit specificity. J Biol Chem 1997; 272: 31092-31099.

20. Nishiyama K, Ogawa H, Yasue H, Soejima H, Misumi K, Takazoe $\mathrm{K}$, et al. Simultaneous elevation of the levels of circulating monocyte chemoattractant protein- 1 and tissue factor in acute coronary syndromes. Jpn Circ J 1998; 62: 710-712.

21. Hojo Y, Ikeda U, Takahashi M, Shimada K. Increased levels of monocyte-related cytokines in patients with unstable angina. Atherosclerosis 2002; 161: 403-408.

22. Schumacher M, Halwachs G, Tatzber F, Fruhwald FM, Zweiker R, Watzinger N, et al. Increased neopterin in patients with chronic and acute coronary syndromes. J Am Coll Cardiol 1997; 30: 703-707.

23. Gupta S, Fredericks S, Schwartzman RA, Holt DW, Kaski JC. Serum neopterin in acute coronary syndromes. Lancet 1997; 349: 12521253.

24. Melichar B, Gregor J, Solichova D, Lukes J, Tichy M, Pidrman V. Increased urinary neopterin in acute myocardial infarction. Clin Chem 1994; 40: 338-339.

25. Moreno PR, Falk E, Palacios IF, Newell JB, Fuster V, Fallon JT. Macrophage infiltration in acute coronary syndromes: Implications for plaque rupture. Circulation 1994; 90: 775-778.

26. Falk E. Morphological features of unstable atherothrombotic plaques underlying acute coronary syndromes. Am J Cardiol 1989; 63: $114 \mathrm{E}-120 \mathrm{E}$.

27. Serneri GG, Abbate R, Gori AM, Attanasio M, Martini F, Giusti B, et al. Transient intermittent lymphocyte activation is responsible for the instability of angina. Circulation 1992; 86: 790-797.

28. Neri Serneri GG, Prisco D, Martini F, Gori AM, Brunelli T, Poggesi $\mathrm{L}$, et al. Acute T-cell activation is detectable in unstable angina. Circulation 1997; 95: 1806-1812.

29. Libby P. Molecular bases of the acute coronary syndromes. Circulation 1995; 91: 2844-2850.

30. Oshima S, Ogawa H, Hokimoto S, Nakamura S, Noda K, Saito T, et al. Plasma monocyte chemoattractant protein-1 antigen levels and the risk of restenosis after coronary stent implntation. Jpn Circ J 2001; 65: 261-264.

31. Hokimoto S, Oike Y, Saito T, Kitaoka M, Oshima S, Noda K, et al. Increased expression of monocyte chemoattractant protein-1 in atherectomy specimens from patients with restenosis after percutaneous transluminal coronary angioplasty. Circ J 2002; 66: 114-116. 